\title{
Mean-field theory of the Kondo effect in quantum dots with an even number of electrons
}

\author{
Mikio Eto ${ }^{1,2}$ and Yuli V. Nazarov ${ }^{1}$ \\ ${ }^{1}$ Department of Applied Physics/DIMES, Delft University of Technology, Lorentzweg 1, 2628 CJ Delft, The Netherlands \\ ${ }^{2}$ Faculty of Science and Technology, Keio University, 3-14-1 Hiyoshi, Kohoku-ku, Yokohama 223-8522, Japan
}

(Received 9 January 2001; published 8 August 2001)

\begin{abstract}
We investigate the enhancement of the Kondo effect in quantum dots with an even number of electrons, using a scaling method and a mean field theory. We evaluate the Kondo temperature $T_{\mathrm{K}}$ as a function of the energy difference between spin-singlet and -triplet states in the dot, $\Delta$, and the Zeeman splitting, $E_{\mathrm{Z}}$. If the Zeeman splitting is small, $E_{\mathrm{Z}} \ll T_{\mathrm{K}}$, the competition between the singlet and triplet states enhances the Kondo effect. $T_{\mathrm{K}}$ reaches its maximum around $\Delta=0$ and decreases with $\Delta$ obeying a power law. If the Zeeman splitting is strong, $E_{\mathrm{Z}} \gg T_{\mathrm{K}}$, the Kondo effect originates from the degeneracy between the singlet state and one of the components of the triplet state at $-\Delta \sim E_{\mathrm{Z}}$. We show that $T_{\mathrm{K}}$ exhibits another power-law dependence on $E_{\mathrm{Z}}$. The mean field theory provides a unified picture to illustrate the crossover between these regimes. The enhancement of the Kondo effect can be understood in terms of the overlap between the Kondo resonant states created around the Fermi level. These resonant states provide the unitary limit of the conductance $G \sim 2 e^{2} / h$.
\end{abstract}

DOI: 10.1103/PhysRevB.64.085322

PACS number(s): 73.23.Hk, 72.15.Qm, 85.35.Be

\section{INTRODUCTION}

The Kondo effect observed in semiconductor quantum dots has attracted a lot of interest. ${ }^{1-5}$ In a quantum dot, the number of electrons $N$ is fixed by the Coulomb blockade to integer values and can be tuned by the gate voltage. Usually the discrete spin-degenerate levels in the quantum dot are consecutively occupied, and the total spin is zero or $1 / 2$ for an even and odd number of electrons, respectively. The Kondo effect takes place only in the latter case. The spin $1 / 2$ in the dot is coupled to the Fermi sea in external leads through tunnel barriers, which results in the formation of the Kondo resonant state at the Fermi level. ${ }^{6-8}$ The conductance through the dot is enhanced to a value of the order of $e^{2} / h$ at low temperatures of $T \ll T_{\mathrm{K}}$ (Kondo temperature). ${ }^{9-13}$ This is called the unitary limit. When $N$ is even, there is no localized spin and thus the Kondo effect is not relevant.

Recently Sasaki et al. has found a large Kondo effect in so-called "vertical" quantum dots with an even $N .{ }^{14}$ The spacing of discrete levels in such dots is comparable with the strength of electron-electron Coulomb interaction. Hence the electronic states deviate from the simple picture mentioned above. ${ }^{15,16}$ If two electrons are put into nearly degenerate levels, the exchange interaction favors a spin triplet (Hund's rule). ${ }^{15}$ This state is changed to a spin singlet by applying a magnetic field perpendicularly to the dots, which increases the level spacing. Hence the energy difference between the singlet and triplet states, $\Delta$, can be controlled experimentally by the magnetic field. The Kondo effect is significantly enhanced around the degeneracy point between the triplet and singlet states, $\Delta=0$. Tuning of the energy difference between the spin states is hardly possible in traditional Kondo systems of dilute magnetic impurities in metal and thus this situation is quite unique to the quantum dot systems.

The Kondo effect in multilevel quantum dots has been investigated theoretically by several groups. ${ }^{17-20}$ They have shown that the contribution from multilevels enhances the Kondo effect. In our previous paper, ${ }^{21}$ we have considered the experimental situation by Sasaki et al. in which the spin- singlet and -triplet states are almost degenerate. We have calculated the Kondo temperature $T_{\mathrm{K}}$ as a function of $\Delta$, using the "poor man's" scaling method. ${ }^{22-24}$ We have shown that $T_{\mathrm{K}}(\Delta)$ is maximal around $\Delta=0$ and decreases with increasing $\Delta$ obeying a power law, $T_{\mathrm{K}}(\Delta) \propto 1 / \Delta^{\gamma}$. The exponent $\gamma$ is not universal but depends on a ratio of the initial coupling constants. Our results indicate that the Kondo effect is enhanced by the competition between singlet and triplet states, in agreement with the experimental findings. ${ }^{14}$

We have disregarded the Zeeman splitting of the spintriplet state, $-E_{\mathrm{Z}} M(M=0, \pm 1$ is $z$ component of the total spin $S=1$ ), since this is a small energy scale in the experimental situation, $E_{\mathrm{Z}} \ll T_{\mathrm{K}} \cdot{ }^{14}$ Pustilnik et al. have studied another situation where the Zeeman effect is relevant, $E_{\mathrm{Z}}$ $\gg T_{\mathrm{K}} \cdot{ }^{25}$ They have considered "lateral" quantum dots with an even $N$, when the ground state is a spin singlet and the first excited state is a triplet $(\Delta<0)$. By applying a quite large magnetic field parallel to the dots, the Zeeman effect reduces the energy of one component of the triplet state, $|S M\rangle=|11\rangle$, and finally makes it the ground state. At the critical magnetic field of $E_{\mathrm{Z}}=-\Delta$, the energy of the state $|11\rangle$ is matched with that of the singlet state, $|00\rangle$. They have found that a Kondo effect arises from the degeneracy between the two states. This is contrast to the usual case with spin $1 / 2$, in which the Zeeman effect lifts off the degeneracy of the spin states and, as a result, breaks the Kondo effect. A similar idea has been proposed by Giuliano and Tagliacozzo. ${ }^{26}$ Their mechanism might explain some experimental results of the Kondo effect in quantum dots under high magnetic fields. ${ }^{4,27}$ Indeed, this type of Kondo effect has been reported in carbon nanotubes where the Zeeman effect is stronger than in semiconductor heterostructures. ${ }^{28}$

The purpose of the present paper is to construct a general theory for the enhancement of the Kondo effect in quantum dots with an even number of electrons, with changing $\Delta$ and $E_{\mathrm{Z}}$. Hence various experimental situations are analyzed in a unified way. We adopt the poor man's scaling method along with the mean field theory. It is well known that the characteristic energy scale of Kondo physics, the Kondo tempera- 
ture $T_{\mathrm{K}}$, is determined by all the energies from $T_{\mathrm{K}}$ up to the upper cutoff. ${ }^{7,8}$ By the scaling method, we can evaluate $T_{\mathrm{K}}$ (its exponential part at least) by taking all the energies properly. ${ }^{22-24}$ When $E_{\mathrm{Z}}$ is negligible, the energies from $\Delta$ to the upper cutoff would feel fourfold degeneracy of the dot states, $|1 M\rangle(M=0, \pm 1)$ and $|00\rangle$, which enhances the Kondo temperature. With increasing $\Delta, T_{\mathrm{K}}$ decreases by a power law. ${ }^{21}$ We extend our previous calculations to the case of $E_{\mathrm{Z}}=-\Delta \gg T_{\mathrm{K}}$ which has been discussed by Pustilnik et al. ${ }^{25}$ and Giuliano and Tagliacozzo. ${ }^{26}$ We take into account the energies not only from $T_{\mathrm{K}}$ to $E_{\mathrm{Z}}$, where only two degenerate states $|11\rangle$ and $|00\rangle$ are relevant, but also from $E_{Z}$ to the upper cutoff, where the dot states seem fourfold degenerate. The latter energy region has been neglected in Refs. 25 and 26. In consequence we find a power-law dependence of $T_{\mathrm{K}}$ on $E_{\mathrm{Z}}$ again.

The mean field theory of the Kondo effect was pioneered by Yoshimori and Sakurai ${ }^{29}$ and is commonly used for the Kondo lattice model. ${ }^{30}$ It is useful to capture main qualitative features of the Kondo effect; renormalizability at the scale of $T_{\mathrm{K}}$, resonances at the Fermi level, and resonant transmission. The simplicity and universality of the mean field theory have driven us to apply it to the problem in question. Generally the Kondo effect gives rise to a many-body ground state which consists of the dot states $|S M\rangle=f_{S M}^{\dagger}|0\rangle$ and the conduction electrons $\Pi c_{k \sigma}^{\dagger}|0\rangle$. The total spin of this ground state is less than the original spin $S$ localized in the dot. The binding energy is of the order of the Kondo temperature $T_{\mathrm{K}}$. We take into account the spin couplings between the dot states and conduction electrons, $\left\langle f_{S M}^{\dagger} c_{k \sigma}\right\rangle$, by the mean field, neglecting their fluctuations. ${ }^{31}$ These spin couplings give rise to resonant states around the Fermi level $\mu$ with the width of the order of $T_{\mathrm{K}}$. The conduction electrons can be transported through the resonant levels, which yields the unitary limit of the conductance $G \sim 2 e^{2} / h$. For our study, the mean field calculations have the following advantages. (i) The enhancement of $T_{\mathrm{K}}$ by the competition between the singlet and triplet states can be directly understood in terms of the overlap between their Kondo resonant states. (ii) The power-law dependence of $T_{\mathrm{K}}$ on $\Delta$ or $E_{\mathrm{Z}}$ is obtained, which is in accordance with the calculated results by the scaling method. (iii) The mean field calculations are applicable to the intermediate regions where two of $T_{\mathrm{K}}, \Delta$, and $E_{\mathrm{Z}}$, are of the same order. The poor man's scaling method hardly gives any results in these regions. Hence we can examine the whole parameter region of $\Delta$ and $E_{\mathrm{Z}}$ by the mean field theory. The disadvantage of the mean field calculations is that they only give qualitative answers. ${ }^{31}$ Hence the mean field theory and scaling method are complementary to each other for understanding the Kondo effect.

We shall discuss the relation of our approach to the renormalization group analysis of the multilevel Kondo effect. ${ }^{23,24}$ Our model effectively reduces to the one with two channels in the leads and spin-triplet (and -singlet) state in the dot when $E_{\mathrm{Z}} \ll T_{\mathrm{K}}$. The ground state of this model would be believed to be a spin singlet, which corresponds to the full screening of the dot spin. The poor man's scaling approach and our mean field theory, however, show a tendency to the formation of the underscreened Kondo ground state with spin $1 / 2$. We should mention that the exact ground state cannot be determined within the limits of the applicability of these approaches. Pustilnik and Glazman have recently proposed a different model for the "triplet-singlet Kondo effect." ${ }^{, 32}$ In our notation, they set $C_{1}=\sqrt{2}, C_{2}=0$ in Eq. (4) for the singlet state. Their model can be directly mapped onto a special case of the two-impurity Kondo model, ${ }^{33}$ for which the ground state is a spin singlet. We are concerned about the case of $C_{1} \approx C_{2}$, and we find that the difference between $C_{1}$ and $C_{2}$ reduces as a result of the renormalization. ${ }^{21}$ This suggests that the case considered in Ref. 32 is by no means a generic one.

This paper is organized as follows. Our model is presented in the next section. In Sec. III, we rederive $T_{\mathrm{K}}(\Delta)$ when the Zeeman splitting is irrelevant, using the poor man's scaling method, in a simpler form than our previous work. ${ }^{21}$ Then we extend our calculations to the case of $E_{\mathrm{Z}}=-\Delta$ $\gg T_{\mathrm{K}}$. Section IV is devoted to the mean field theory for the Kondo effect in quantum dots. First we explain this theory for the usual Kondo effect in a quantum dot with $S=1 / 2$. Then we apply the mean field scheme to our model with an even number of electrons in the dot. The conclusions and discussion are given in the last section.

\section{MODEL}

We are interested in the competition between the spinsinglet and -triplet states in a quantum dot. To model the situation, it is sufficient to consider two extra electrons in a quantum dot at the background of a singlet state of all other $N-2$ electrons, which we will regard as the vacuum $|0\rangle$. These two extra electrons occupy two levels of different orbital symmetry. ${ }^{34}$ The energies of the levels are $\varepsilon_{1}$ and $\varepsilon_{2}$. Possible two-electron states are (i) the spin-triplet state, (ii) the spin-singlet state of the same orbital symmetry as the triplet state, $1 / \sqrt{2}\left(d_{1 \uparrow}^{\dagger} d_{2 \downarrow}^{\dagger}-d_{1 \downarrow}^{\dagger} d_{2 \uparrow}^{\dagger}\right)|0\rangle$, and (iii) two singlet states of different orbital symmetry, $d_{1 \uparrow}^{\dagger} d_{1 \downarrow}^{\dagger}|0\rangle, d_{2 \uparrow}^{\dagger} d_{2 \downarrow}^{\dagger}|0\rangle$. Among the singlet states, we only consider a state of the lowest energy, which belongs to group (iii). Thus we restrict our attention to four states, $|S M\rangle$ :

$$
\begin{gathered}
|11\rangle=d_{1 \uparrow}^{\dagger} d_{2 \uparrow}^{\dagger}|0\rangle, \\
|10\rangle=\frac{1}{\sqrt{2}}\left(d_{1 \uparrow}^{\dagger} d_{2 \downarrow}^{\dagger}+d_{1 \downarrow}^{\dagger} d_{2 \uparrow}^{\dagger}\right)|0\rangle, \\
|1-1\rangle=d_{1 \downarrow}^{\dagger} d_{2 \downarrow}^{\dagger}|0\rangle, \\
|00\rangle=\frac{1}{\sqrt{2}}\left(C_{1} d_{1 \uparrow}^{\dagger} d_{1 \downarrow}^{\dagger}-C_{2} d_{2 \uparrow}^{\dagger} d_{2 \downarrow}^{\dagger}\right)|0\rangle,
\end{gathered}
$$

where $d_{i \sigma}^{\dagger}$ creates an electron with spin $\sigma$ in level $i$. The coefficients in the singlet state, $C_{1}$ and $C_{2}\left(\left|C_{1}\right|^{2}+\left|C_{2}\right|^{2}\right.$ $=2$ ), are determined by the electron-electron interaction and one-electron level spacing $\delta=\varepsilon_{2}-\varepsilon_{1}$. We set $C_{1}=C_{2}=1$. This is the case for $\delta=0 .{ }^{35}$ Although $C_{1} \neq C_{2}$ in general, the scaling analysis shows that the Kondo temperature is the 
(a)

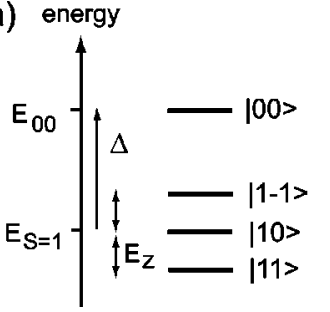

(b)

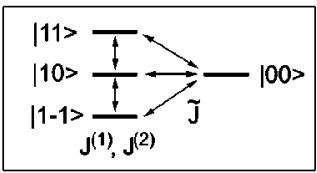

FIG. 1. (a) The energy diagram for the spin states $|S M\rangle$ considered in our model. $\Delta=E_{00}-E_{S=1}$ and $E_{\mathrm{Z}}$ is the Zeeman splitting. (b) Spin-flip processes between the spin states. The exchange couplings $J^{(i)}$ involving the spin-triplet state only are accompanied by the scattering of conduction electrons of channel $i$. Those involving both the spin-triplet and -singlet states, $\widetilde{J}$, are accompanied by the interchannel scattering of conduction electrons.

same as that in the case of $C_{1}=C_{2}=1$, apart from a prefactor. ${ }^{21}$ The energies of the triplet state are given by

$$
E_{S=1, M}=E_{S=1}-E_{Z} M,
$$

and the energy of the singlet state is denoted by $E_{00}$. We define $\Delta$ by

$$
\Delta=E_{00}-E_{S=1} .
$$

The energy diagram for the spin states is indicated in Fig. $1(\mathrm{a})$.

The dot is connected to two external leads $L, R$ with free electrons being described by

$$
H_{\text {leads }}=\sum_{\alpha=L, R} \sum_{k \sigma i} \varepsilon_{k}^{(i)} c_{\alpha, k \sigma}^{(i) \dagger} c_{\alpha, k \sigma}^{(i)},
$$

where $c_{\alpha, k \sigma}^{(i) \dagger}\left(c_{\alpha, k \sigma}^{(i)}\right)$ is the creation (annihilation) operator of an electron in lead $\alpha$ with momentum $k$, spin $\sigma$, and orbital symmetry $i(=1,2)$. The density of states $\nu$ in the leads remains constant in the energy band of $[-D, D]$. The tunneling between the dot and the leads is written as

$$
H_{T}=\sum_{\alpha=L, R} \sum_{k \sigma i}\left(V_{\alpha, i} c_{\alpha, k \sigma}^{(i) \dagger} d_{i \sigma}+\text { H.c. }\right) .
$$

We assume that the orbital symmetry is conserved in the tunneling processes. ${ }^{34}$ To avoid the complication due to the fact that there are two leads $\alpha=L, R$, we perform a unitary transformation for electron modes in the leads along the lines of Ref. 9; $\quad c_{k \sigma}^{(i)}=\left(V_{L, i}^{*} c_{L, k \sigma}^{(i)}+V_{R, i}^{*} c_{R, k \sigma}^{(i)}\right) / V_{i}, \quad \bar{c}_{k \sigma}^{(i)}$ $=\left(-V_{R, i} c_{L, k \sigma}^{(i)}+V_{L, i} c_{R, k \sigma}^{(i)}\right) / V_{i}$, with $V_{i}=\sqrt{\left|V_{L, i}\right|^{2}+\left|V_{R, i}\right|^{2}}$. The modes $\bar{c}_{k \sigma}^{(i)}$ are not coupled to the quantum dot and shall be disregarded hereafter. Then $H_{\text {leads }}$ and $H_{T}$ are rewritten as

$$
\begin{gathered}
H_{\text {leads }}=\sum_{k \sigma i} \varepsilon_{k}^{(i)} c_{k \sigma}^{(i) \dagger} c_{k \sigma}^{(i)}, \\
H_{T}=\sum_{k \sigma i} V_{i}\left(c_{k \sigma}^{(i) \dagger} d_{i \sigma}+\text { H.c. }\right) .
\end{gathered}
$$

We assume that the state of the dot with $N$ electrons is stable, so that addition/extraction energies, $E^{ \pm} \equiv E(N \pm 1)$

$-E(N) \mp \mu$, where $\mu$ is the Fermi energy in the leads, are positive. We are interested in the case where $E^{ \pm} \gg|\Delta|, \delta$ and also exceed the level broadening $\Gamma^{i}=\pi \nu V_{i}^{2}$ and temperature $T$ (Coulomb blockade region). In this case we can integrate out the states with one or three extra electrons. This is equivalent to Schrieffer-Wolff transformation that is used to obtain the conventional Kondo model. ${ }^{7,8}$ We obtain the following effective low-energy Hamiltonian

$$
H_{\text {eff }}=H_{\text {leads }}+H_{\text {dot }}+H^{S=1}+H^{S=1 \leftrightarrow 0}+H_{\text {eff }}^{\prime} .
$$

The Hamiltonian of the dot $H_{\mathrm{dot}}$ reads

$$
H_{\mathrm{dot}}=\sum_{S, M} E_{S M} f_{S M}^{\dagger} f_{S M},
$$

using pseudofermion operators $f_{S M}^{\dagger}\left(f_{S M}\right)$ which create (annihilate) the state $|S M\rangle$. The condition of

$$
\sum_{S M} f_{S M}^{\dagger} f_{S M}=1
$$

should be fulfilled. The third term $H^{S=1}$ represents the spinflip processes among three components of the spin-triplet state. This resembles a conventional Kondo Hamiltonian for $S=1$ in terms of the spin operator $\hat{S}$ :

$$
\begin{aligned}
H^{S=1}= & \sum_{k k^{\prime}} \sum_{i=1,2} J^{(i)}\left[\hat{S}_{+} c_{k^{\prime} \downarrow}^{(i) \dagger} c_{k \uparrow}^{(i)}+\hat{S}_{-} c_{k^{\prime} \uparrow}^{(i) \dagger} c_{k \downarrow}^{(i)}+\hat{S}_{z}\left(c_{k^{\prime} \uparrow}^{(i) \dagger} c_{k \uparrow}^{(i)}\right.\right. \\
& \left.\left.-c_{k^{\prime} \downarrow}^{(i) \dagger} c_{k \downarrow}^{(i)}\right)\right] \\
= & \sum_{k k^{\prime}} \sum_{i=1,2} J^{(i)}\left[\sqrt{2}\left(f_{11}^{\dagger} f_{10}+f_{10}^{\dagger} f_{1-1}\right) c_{k^{\prime} \downarrow}^{(i) \dagger} c_{k \uparrow}^{(i)}\right. \\
& +\sqrt{2}\left(f_{10}^{\dagger} f_{11}+f_{1-1}^{\dagger} f_{10}\right) c_{k^{\prime} \uparrow}^{(i) \dagger} c_{k \downarrow}^{(i)}+\left(f_{11}^{\dagger} f_{11}-f_{1-1}^{\dagger} f_{1-1}\right) \\
& \left.\times\left(c_{k^{\prime} \uparrow}^{(i) \dagger} c_{k \uparrow}^{(i)}-c_{k^{\prime} \downarrow}^{(i) \dagger} c_{k \downarrow}^{(i)}\right)\right] .
\end{aligned}
$$

The exchange coupling $J^{(i)}$ is accompanied by the scattering of conduction electrons of channel $i$. The fourth term $H^{S=1 \leftrightarrow 0}$ in $H_{\text {eff }}$ describes the conversion between the spintriplet and -singlet states accompanied by the interchannel scattering of conduction electrons

$$
\begin{aligned}
H^{S=1 \leftrightarrow 0}= & \sum_{k k^{\prime}} \widetilde{J}\left[\sqrt{2}\left(f_{11}^{\dagger} f_{00}-f_{00}^{\dagger} f_{1-1}\right) c_{k^{\prime} \downarrow}^{(1) \dagger} c_{k \uparrow}^{(2)}+\sqrt{2}\left(f_{00}^{\dagger} f_{11}\right.\right. \\
& \left.-f_{1-1}^{\dagger} f_{00}\right) c_{k^{\prime} \uparrow}^{(1) \dagger} c_{k \downarrow}^{(2)}-\left(f_{10}^{\dagger} f_{00}+f_{00}^{\dagger} f_{10}\right)\left(c_{k^{\prime} \uparrow}^{(1) \dagger} c_{k \uparrow}^{(2)}\right. \\
& \left.\left.-c_{k^{\prime} \downarrow}^{(1) \dagger} c_{k \downarrow}^{(2)}\right)+(1 \leftrightarrow 2)\right] .
\end{aligned}
$$

The coupling constants are given by

$$
\begin{gathered}
J^{(i)}=\frac{V_{i}^{2}}{2 E_{\mathrm{c}}}, \\
\widetilde{J}=\frac{V_{1} V_{2}}{2 E_{\mathrm{c}}},
\end{gathered}
$$


where $1 / E_{\mathrm{c}}=1 / E^{+}+1 / E^{-}$. Note that $\widetilde{J}^{2}=J^{(1)} J^{(2)}$. The last term $H_{\text {eff }}^{\prime}$ represents the scattering processes of conduction electrons without any change of the dot state and is not relevant for the current discussion. The spin-flip processes included in our model are shown in Fig. 1(b).

\section{SCALING CALCULATIONS}

In this section we calculate the Kondo temperature $T_{\mathrm{K}}$ using the poor man's scaling technique. ${ }^{22-24}$ By changing the energy scale (bandwidth of the conduction electrons) from $D$ to $D-|d D|$, we obtain the scaling equations using the second-order perturbation calculations with respect to the exchange couplings, $J^{(1)}, J^{(2)}$, and $\widetilde{J}$. With decreasing $D$, the exchange couplings are renormalized. The Kondo temperature is determined as the energy scale at which the exchange couplings become so large that the perturbation breaks down.

\section{A. In the absence of Zeeman effect}

When the Zeeman splitting is small and irrelevant, $E_{\mathrm{Z}}$ $\ll T_{\mathrm{K}}$, we obtain a closed form of the scaling equations for $J^{(1)}, J^{(2)}$, and $\widetilde{J}$ in two limits. ${ }^{21}$ (i) When the energy scale $D$ is much larger than the energy difference $|\Delta|, H_{\text {dot }}$ can be safely disregarded in $H_{\text {eff }}$. The scaling equations can be written as

$$
\frac{d}{d \ln D}\left(\begin{array}{cc}
J^{(1)} & \widetilde{J} \\
\widetilde{J} & J^{(2)}
\end{array}\right)=-2 \nu\left(\begin{array}{cc}
J^{(1)} & \widetilde{J} \\
\widetilde{J} & J^{(2)}
\end{array}\right)^{2} .
$$

(ii) For $D \ll \Delta$, the ground state of the dot is a spin triplet and the singlet state can be disregarded. Then $J^{(1)}$ and $J^{(2)}$ evolve independently,

$$
\frac{d}{d \ln D} J^{(i)}=-2 \nu J^{(i) 2},
$$

whereas $\widetilde{J}$ does not change.

In the case of $|\Delta| \ll T_{\mathrm{K}}$, the scaling equations (16) remain valid till the scaling ends. The matrix in Eq. (16) has eigenvalues of

$$
J_{ \pm}=\left(J^{(1)}+J^{(2)}\right) / 2 \pm \sqrt{\left(J^{(1)}-J^{(2)}\right)^{2} / 4+\widetilde{J}^{2}}=J^{(1)}+J^{(2)}, 0 .
$$

The larger one, $J_{+}$, diverges upon decreasing the bandwidth $D$ and determines $T_{\mathrm{K}}$ :

$$
T_{\mathrm{K}}(0)=D_{0} \exp \left[-1 / 2 \nu J_{+}\right]=D_{0} \exp \left[-1 / 2 \nu\left(J^{(1)}+J^{(2)}\right)\right] .
$$

Here $D_{0}$ is the initial bandwidth, which is given by $\sqrt{E^{+} E^{-}} .36$

When $\Delta>D_{0}$, the scaling equations (17) work in the whole scaling region. This yields

$$
T_{\mathrm{K}}(\infty)=D_{0} \exp \left[-1 / 2 \nu J^{(1)}\right]
$$

for $J^{(1)} \geqslant J^{(2)}$. This is the Kondo temperature for spin-triplet localized spins. ${ }^{37}$
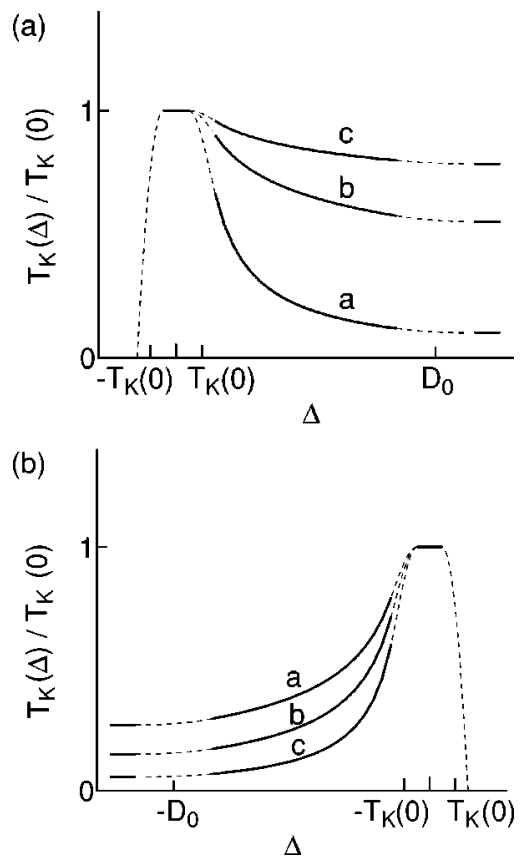

FIG. 2. The scaling calculations of the Kondo temperature $T_{\mathrm{K}}$ as a function of $\Delta$, (a) when the Zeeman splitting is irrelevant, $E_{\mathrm{Z}}$ $\ll T_{\mathrm{K}}$, and (b) in a case of $E_{\mathrm{Z}}=-\Delta . D_{0}$ is the bandwidth in the leads. In both the figures, curve $a, \theta / \pi=0.25$; curve $b, 0.15$; and curve $c, 0.10$, where $\tan ^{2} \theta=J^{(2)} / J^{(1)}$.

In the intermediate region of $T_{\mathrm{K}}(0) \ll \Delta \ll D_{0}$, the exchange couplings develop by Eq. (16) for $D \gg \Delta$. Around $D$ $=\Delta, \widetilde{J}$ saturates while $J^{(1)}$ and $J^{(2)}$ continue to grow with decreasing $D$, following Eq. (17) for $D \ll \Delta$. We match the solutions of these scaling equations at $D \simeq \Delta$ and obtain a power law of $T_{\mathrm{K}}(\Delta)$

$$
T_{\mathrm{K}}(\Delta)=T_{\mathrm{K}}(0)\left[T_{\mathrm{K}}(0) / \Delta\right]^{\tan ^{2} \theta},
$$

with

$\tan \theta=\widetilde{J} /\left[\sqrt{\left(J^{(1)}-J^{(2)}\right)^{2} / 4+\widetilde{J}^{2}}+\left(J^{(1)}-J^{(2)}\right) / 2\right]=\sqrt{J^{(2)} / J^{(1)}}$

for $J^{(1)} \geqslant J^{(2)}$. Here $(\cos \theta, \sin \theta)^{T}$ is the eigenfunction of the matrix in Eq. (16) corresponding to $J_{+} \cdot \theta \sim 0$ for $J^{(1)}$ $\gg J^{(2)}$ and $\theta=\pi / 4$ for $J^{(1)}=J^{(2)}$. In general, $0<\theta \leqslant \pi / 4$ and thus $0<\tan ^{2} \theta \leqslant 1$.

Finally, for $\Delta<0$, all the coupling constants saturate and no Kondo effect is expected, provided $|\Delta| \gg T_{\mathrm{K}}(0)$. Thus $T_{\mathrm{K}}$ quickly decreases to zero at $\Delta \sim-T_{\mathrm{K}}(0)$. The Kondo temperature as a function of $\Delta$ is schematically shown in Fig. 2(a).

\section{B. Case of $E_{\mathrm{Z}}=-\Delta$}

When $E_{\mathrm{Z}}=-\Delta$, the energies of states $|00\rangle$ and $|11\rangle$ are degenerate. Then the Kondo effect is expected even when $|\Delta| \gg T_{\mathrm{K}}(0){ }^{25,26}$ In this subsection we evaluate $T_{\mathrm{K}}$ in the special case of $E_{\mathrm{Z}}=-\Delta$ by the poor man's scaling method.

(i) For the energy scale of $D \gg|\Delta|=E_{\mathrm{Z}}, H_{\text {dot }}$ can be disregarded in $H_{\text {eff }}$. The exchange couplings, $J^{(1)}, J^{(2)}$, and $\widetilde{J}$, 
evolve following Eq. (16). (ii) In another limit of $D \ll|\Delta|$ $=E_{\mathrm{Z}}$, only the states $|00\rangle$ and $|11\rangle$ are relevant. In $H_{\text {eff }}$,

$$
\begin{aligned}
H_{\mathrm{eff}}^{|00\rangle,|11\rangle}= & \sum_{k k^{\prime}} \sum_{i=1,2}\left[\frac { 1 } { 2 } J _ { \mathrm { s } } ^ { ( i ) } ( f _ { 1 1 } ^ { \dagger } f _ { 1 1 } - f _ { 0 0 } ^ { \dagger } f _ { 0 0 } ) \left(c_{k^{\prime} \uparrow}^{(i) \dagger} c_{k \uparrow}^{(i)}\right.\right. \\
& \left.-c_{k^{\prime} \downarrow}^{(i) \dagger} c_{k \downarrow}^{(i)}\right)+\frac{1}{2} J_{\mathrm{c}}^{(i)}\left(f_{11}^{\dagger} f_{11}+f_{00}^{\dagger} f_{00}\right)\left(c_{k^{\prime} \uparrow}^{(i) \dagger} c_{k^{\prime} \uparrow}^{(i)}\right. \\
& \left.\left.-c_{k^{\prime} \downarrow}^{(i) \dagger} c_{k \downarrow}^{(i)}\right)\right]+\sum_{k k^{\prime}} \sqrt{2} \widetilde{J}\left[f_{11}^{\dagger} f_{00} c_{k^{\prime} \downarrow}^{(1) \dagger} c_{k \uparrow}^{(2)}\right. \\
& \left.+f_{00}^{\dagger} f_{11} c_{k^{\prime} \uparrow}^{(1) \dagger} c_{k \downarrow}^{(2)}+(1 \leftrightarrow 2)\right] .
\end{aligned}
$$

$J_{\mathrm{s}}^{(i)}=J_{\mathrm{c}}^{(i)}=J^{(i)}$ initially. The scaling procedure yields

$$
\begin{gathered}
\frac{d}{d \ln D} J_{\mathrm{s}}^{(i)}=-4 \nu \widetilde{J}^{2}, \\
\frac{d}{d \ln D} \widetilde{J}=-\nu\left(J_{\mathrm{s}}^{(1)}+J_{\mathrm{s}}^{(2)}\right) \widetilde{J},
\end{gathered}
$$

and $J_{\mathrm{c}}^{(i)}$ do not change. These scaling equations are nearly equivalent to those of the anisotropic Kondo model with $S$ $=1 / 2,{ }^{22}$ as pointed out in Refs. 25,26 .

When $|\Delta|=E_{\mathrm{Z}}>D_{0}$, the scaling equations (24) remain valid in the whole scaling region. This yields the Kondo temperature

$$
T_{\mathrm{K}}(\infty)=D_{0} \exp \left[-A(\theta) / 2 \nu\left(J^{(1)}+J^{(2)}\right)\right]
$$

with

$$
A(\theta)= \begin{cases}\frac{1}{\lambda} \ln \left(\frac{1+\lambda}{1-\lambda}\right) & (0<\theta \leqslant \pi / 8) \\ \frac{2}{\lambda} \tan ^{-1} \lambda & (\pi / 8<\theta \leqslant \pi / 4),\end{cases}
$$

where $\lambda=\sqrt{|\cos 4 \theta|} .^{38} A(\theta)$ decreases monotonically with increasing $\theta . A(\theta) \rightarrow \infty$ as $\theta \rightarrow 0 . A(\pi / 8)=2$ and $A(\pi / 4)$ $=\pi / 2$. When $J^{(1)}+J^{(2)}$ is fixed, $T_{\mathrm{K}}(\infty)$ is the largest at $J^{(1)}=J^{(2)}(\theta=\pi / 4)$ and becomes smaller with decreasing $J^{(2)} / J^{(1)}\left(=\tan ^{2} \theta\right)$.

In the intermediate region, $T_{\mathrm{K}}(0) \ll|\Delta|=E_{\mathrm{Z}} \ll D_{0}$, we match the solutions of Eqs. (16) and (24) at $D \simeq|\Delta|$. We obtain a power law

$$
T_{\mathrm{K}}(\Delta)=T_{\mathrm{K}}(0)\left[T_{\mathrm{K}}(0) /|\Delta|\right]^{A(\theta)-1} .
$$

Figure 2(b) shows the behaviors of $T_{\mathrm{K}}(\Delta)$ in the case of $E_{\mathrm{Z}}=-\Delta$.

\section{MEAN FIELD CALCULATIONS}

\section{A. Kondo resonance for $\operatorname{spin} S=1 / 2$}

To illustrate the mean field theory for the Kondo effect in quantum dots, we begin with the usual case of $S=1 / 2$. We assume that one level $\left(E_{0}\right)$ in a quantum dot is occupied by an electron with spin either up or down $(\sigma=\uparrow, \downarrow)$. The effective low-energy Hamiltonian is

$$
H=\sum_{k \sigma} \varepsilon_{k} c_{k \sigma}^{\dagger} c_{k \sigma}+\sum_{\sigma} E_{\sigma} f_{\sigma}^{\dagger} f_{\sigma}+J \sum_{k k^{\prime}} \sum_{\sigma, \sigma^{\prime}} f_{\sigma}^{\dagger} f_{\sigma^{\prime}} c_{k^{\prime} \sigma^{\prime}}^{\dagger} c_{k \sigma},
$$

with the constraint of

$$
f_{\uparrow}^{\dagger} f_{\uparrow}+f_{\downarrow}^{\dagger} f_{\downarrow}=1 .
$$

For electrons in leads $L, R$, we have performed a unitary transformation of $c_{k \sigma}=\left(V_{L}^{*} c_{L, k \sigma}+V_{R}^{*} c_{R, k \sigma}\right) / \sqrt{\left|V_{L}\right|^{2}+\left|V_{R}\right|^{2}}$ where $V_{\alpha}$ is the tunneling coupling to lead $\alpha .{ }^{9}$ The last term in Eq. (28) represents the exchange coupling between $S$ $=1 / 2$ in the dot and conduction electrons (see Appendix A).

In the mean field theory, we introduce the order parameter

$$
\langle\Xi\rangle=\frac{1}{\sqrt{2}} \sum_{k}\left(\left\langle f_{\uparrow}^{\dagger} c_{k \uparrow}\right\rangle+\left\langle f_{\downarrow}^{\dagger} c_{k \downarrow}\right\rangle\right)
$$

to describe the spin couplings between the dot states and conduction electrons. The mean field Hamiltonian reads

$$
\begin{aligned}
H_{\mathrm{MF}}= & \sum_{k \sigma} \varepsilon_{k} c_{k \sigma}^{\dagger} c_{k \sigma}+\sum_{\sigma} E_{\sigma} f_{\sigma}^{\dagger} f_{\sigma}-\sum_{k, \sigma}\left(\sqrt{2} J\langle\Xi\rangle c_{k \sigma}^{\dagger} f_{\sigma}\right. \\
& + \text { H.c. })+2 J|\langle\Xi\rangle|^{2}+\lambda\left(\sum_{\sigma} f_{\sigma}^{\dagger} f_{\sigma}-1\right) .
\end{aligned}
$$

The constraint, Eq. (29), is taken into account by the last term with a Lagrange multiplier $\lambda$. By minimizing the expectation value of $H_{\mathrm{MF}},\langle\Xi\rangle$ is determined self-consistently (Appendix A).

In the absence of the Zeeman effect, $E_{\uparrow}=E_{\downarrow}=E_{0}$. The mean field Hamiltonian, $H_{\mathrm{MF}}$, represents a resonant tunneling through an "energy level," $\widetilde{E}_{0}=E_{0}+\lambda$, with "tunneling coupling," $\widetilde{V}=-\sqrt{2} J\langle\Xi\rangle . \widetilde{V}$ provides a finite width of the resonance, $\widetilde{\Delta}_{0}=\pi \nu|\widetilde{V}|^{2}$, with $\nu$ being the density of states in the leads. The constraint, Eq. (29), requires that the states for the pseudofermions are half-filled, that is, $\widetilde{E}_{0}=\mu$. Hence the Kondo resonant state appears just at the Fermi level $\mu$, as indicated in the inset (A) in Fig. 3(a). The self-consistent calculations give us the resonant width

$$
\widetilde{\Delta}_{0}=\pi \nu|\sqrt{2} J\langle\Xi\rangle|^{2}=D_{0} \exp [-1 / 2 \nu J] .
$$

This is identical to the Kondo temperature $T_{\mathrm{K}}$.

In the presence of the Zeeman splitting, $E_{\uparrow}=E_{0}-E_{\mathrm{Z}}$ and $E_{\downarrow}=E_{0}+E_{\mathrm{Z}}$. Hence the resonant level is split for spin-up and -down electrons, $\widetilde{E}_{\uparrow / \downarrow}=E_{\uparrow / \downarrow}+\lambda$. The constraint, Eq. (29), yields $E_{0}+\lambda=\mu$ [see inset (B) in Fig. 3(a)]. The resonant width $\widetilde{\Delta}$ is determined as

$$
\widetilde{\Delta}^{2}+E_{Z}^{2}=\widetilde{\Delta}_{0}^{2},
$$

where $\widetilde{\Delta}_{0}$ is given by Eq. (32). The Kondo temperature is evaluated by this width, $T_{\mathrm{K}}\left(E_{\mathrm{Z}}\right)=\widetilde{\Delta}$. $T_{\mathrm{K}}$ decreases with increasing $E_{\mathrm{Z}}$ and disappears at $E_{\mathrm{Z}}=T_{\mathrm{K}}(0)$, as shown in Fig. 3(a).

The conductance $G$ through the dot is expressed, using $\Gamma_{\alpha}=\pi \nu\left|V_{\alpha}\right|^{2}$, as 

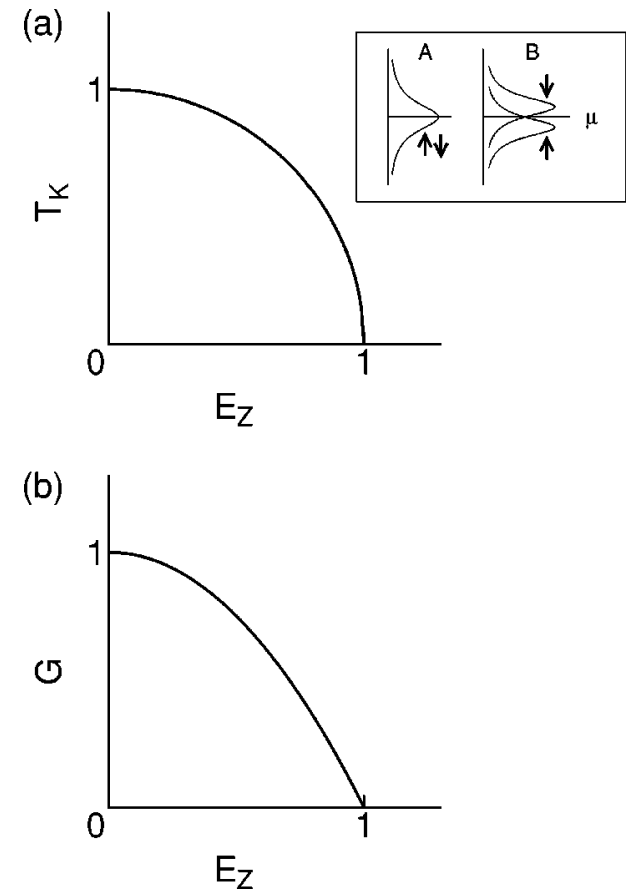

FIG. 3. The mean field calculations for the Kondo effect in a quantum dot with $S=1 / 2$. (a) The Kondo temperature $T_{\mathrm{K}}$ and (b) conductance through the dot, $G$, as functions of the Zeeman splitting $E_{\mathrm{Z}} \cdot T_{\mathrm{K}}$ and $E_{\mathrm{Z}}$ are in units of $D_{0} \exp (-1 / 2 \nu J)$ and $G$ is in units of $\left(2 e^{2} / h\right) 4 \Gamma_{L} \Gamma_{R} /\left(\Gamma_{L}+\Gamma_{R}\right)^{2}$. Inset in (a): The Kondo resonant states created around the Fermi level $\mu$ in the leads, (A) in the absence and (B) presence of the Zeeman splitting. The resonant width is given by $T_{\mathrm{K}}$.

$$
G=\frac{2 e^{2}}{h} \frac{4 \Gamma_{L} \Gamma_{R}}{\left(\Gamma_{L}+\Gamma_{R}\right)^{2}}\left[1-\left(\frac{E_{\mathrm{Z}}}{T_{\mathrm{K}}(0)}\right)^{2}\right]
$$

This is the conductance in the unitary limit for $E_{Z}=0$. Figure 3(b) presents the $E_{Z}$ dependence of the conductance. With increasing $E_{\mathrm{Z}}$, the splitting between the resonant levels for spin up and down becomes larger. In consequence the amplitude of the Kondo resonance decreases at $\mu$, which reduces the conductance.

\section{B. Kondo resonance in the present model}

Now we apply the mean field theory to our model which has the spin-triplet and -singlet states in a quantum dot. The spin states of the coupling to a conduction electron are $(S$ $=1) \otimes(S=1 / 2)=(S=3 / 2) \oplus(S=1 / 2)$ for the former, and $(S=0) \otimes(S=1 / 2)=(S=1 / 2)$ for the latter (Appendix B). To represent the competition between the triplet and single states, therefore, the order parameter should be a spinor of $S=1 / 2$. It is $\langle\vec{\Xi}\rangle$ where

$$
\vec{\Xi}=\sum_{k}\left(\begin{array}{c}
\cos \varphi\left(\sqrt{2} f_{11}^{\dagger} c_{k \uparrow}^{(1)}+f_{10}^{\dagger} c_{k \downarrow}^{(1)}\right) / \sqrt{3}+(\sin \varphi) f_{00}^{\dagger} c_{k \downarrow}^{(2)} \\
\cos \varphi\left(\sqrt{2} f_{1-1}^{\dagger} c_{k \downarrow}^{(1)}+f_{10}^{\dagger} c_{k \uparrow}^{(1)}\right) / \sqrt{3}-(\sin \varphi) f_{00}^{\dagger} c_{k \uparrow}^{(2)}
\end{array}\right)
$$

for $J^{(1)}>J^{(2)}$. A mode of the largest coupling is taken into account in this approximation. The Hamiltonian reads

$$
\begin{aligned}
H_{\mathrm{MF}}= & H_{\text {lead }}+H_{\mathrm{dot}}-J_{\mathrm{MF}}\left[\left\langle\overrightarrow{\vec{\Xi}^{\dagger}}\right\rangle \vec{\Xi}+\vec{\Xi}^{\dagger}\langle\vec{\Xi}\rangle-|\langle\vec{\Xi}\rangle|^{2}\right] \\
& +\lambda\left(\sum_{S M} f_{S M}^{\dagger} f_{S M}-1\right),
\end{aligned}
$$

where

$$
J_{\mathrm{MF}}=J^{(1)}+\sqrt{J^{(1) 2}+3 \widetilde{J}^{2}}
$$

and

$$
\tan \varphi=\sqrt{3} \widetilde{J} / J_{\mathrm{MF}}
$$

The last term in $H_{\mathrm{MF}}$ considers the restriction of Eq. (11). The expectation value of $H_{\mathrm{MF}}$ is minimized with respect to $|\vec{E}|^{2}$. The Kondo temperature can be estimated by

$$
T_{\mathrm{K}}=\pi \nu\left|J_{\mathrm{MF}}\langle\vec{\Xi}\rangle\right|^{2}
$$

using $\langle\vec{\Xi}\rangle$ determined by the self-consistent calculations (Appendix B).

First let us consider the case in the absence of the Zeeman effect, $E_{1 M}=E_{S=1}$ and $E_{00}=E_{S=1}+\Delta$. The resonant level for the triplet state is threefold degenerate at $\widetilde{E}_{S=1}=E_{S=1}$ $+\lambda$, whereas the resonant level for the singlet state is at $\widetilde{E}_{0}=E_{00}+\lambda$. These levels are separated by the energy $\Delta$. The Lagrange multiplier $\lambda$ is determined to fulfill Eq. (11). Figure 4(a) shows the calculated results of $T_{\mathrm{K}}$ as a function of $\Delta$. Both of $T_{\mathrm{K}}$ and $\Delta$ are in units of $D_{0} \exp \left(-1 / \nu J_{\mathrm{MF}}\right)$. We find that (i) $T_{\mathrm{K}}(\Delta)$ reaches its maximum at $\Delta=0$, (ii) for $\Delta \gg T_{\mathrm{K}}(0), T_{\mathrm{K}}(\Delta)$ obeys a power law

$$
T_{\mathrm{K}}(\Delta) \Delta^{\tan ^{2} \varphi}=\text { const., }
$$

and (iii) for $\Delta<0, T_{\mathrm{K}}$ decreases rapidly with increasing $|\Delta|$ and disappears at $\Delta=\Delta_{\mathrm{c}} \sim-T_{\mathrm{K}}(0)$ :

$$
\Delta_{\mathrm{c}}=-D_{0} \exp \left(-1 / \nu J_{\mathrm{MF}}\right)\left(1+\tan ^{2} \varphi\right)\left(\tan ^{2} \varphi\right)^{-\sin ^{2} \varphi} .
$$

These features are in agreement with the results of the scaling calculations.

The behaviors of $T_{\mathrm{K}}(\Delta)$ can be understood as follows. The inset of Fig. 4(a) schematically shows the Kondo resonant states. The resonance of the triplet state is denoted by solid lines, whereas that of the singlet state is by dotted lines. (A) When $\Delta \gg T_{\mathrm{K}}(0)$, the triplet resonance appears around $\mu$, whereas the singlet resonance is far above $\mu$. (B) With a decrease in $\Delta$, the two resonant states are more overlapped at $\mu$, which raises $T_{\mathrm{K}}$ gradually. This results in a power law of $T_{\mathrm{K}}(\Delta)$, Eq. (40). The largest overlap yields the maximum of $T_{\mathrm{K}}$ at $\Delta=0$. (C) When $\Delta<0$, the singlet and triplet resonances are located below and above $\mu$, respectively, being sharper and farther from each other with increasing $|\Delta|$. Finally the Kondo resonance disappears at $\Delta=\Delta_{\mathrm{c}}$.

The conductance through the dot is given by 

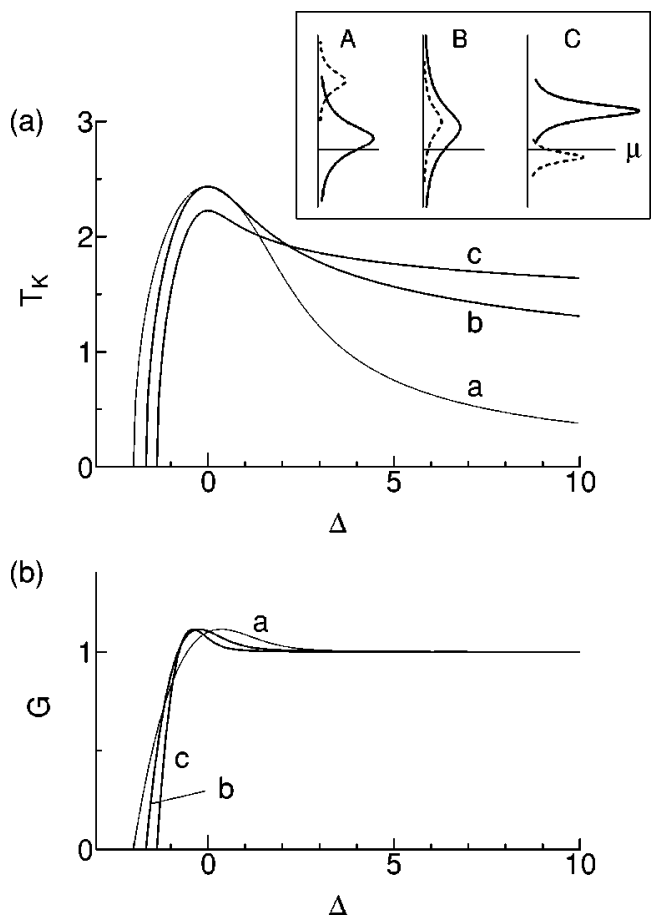

FIG. 4. The mean field calculations for the Kondo effect in the present model. The Zeeman splitting is disregarded $\left(E_{\mathrm{Z}} \ll T_{\mathrm{K}}\right)$. (a) The Kondo temperature $T_{\mathrm{K}}$ and (b) conductance through the dot, $G$, as functions of $\Delta=E_{00}-E_{S=1} . T_{\mathrm{K}}$ and $\Delta$ are in units of $D_{0} \exp \left(-1 / \nu J_{\mathrm{MF}}\right) . G$, in units of $2 e^{2} / h$, is evaluated in a symmetric case of $\Gamma_{L}^{i}=\Gamma_{R}^{i}(i=1,2) . \tan \varphi=\sqrt{3} \widetilde{J} / J_{\mathrm{MF}}$ where curve $a, \varphi / \pi$ $=0.25$; curve $b, 0.15$; and curve $c, 0.10$. Note that $\varphi / \pi \leqslant 1 / 6$ in this approximation (case $a$ is only for reference). Inset in (a): The Kondo resonant states for $S=1$ (solid line) and for $S=0$ (dotted line) when (A) $\Delta \gg T_{\mathrm{K}}(0)$, (B) $\Delta \sim T_{\mathrm{K}}(0)$, and (C) $\Delta<0$.

$$
\begin{aligned}
G /\left(e^{2} / h\right)= & \frac{4 \Gamma_{L}^{1} \Gamma_{R}^{1}}{\left(\Gamma_{L}^{1}+\Gamma_{R}^{1}\right)^{2}}\left(\frac{\widetilde{\Delta}_{11}^{2}}{\left(\varepsilon-\widetilde{E}_{11}\right)^{2}+\widetilde{\Delta}_{11}^{2}}\right. \\
& \left.+\frac{\widetilde{\Delta}_{10}^{2}}{\left(\varepsilon-\widetilde{E}_{10}\right)^{2}+\widetilde{\Delta}_{10}^{2}}\right) \\
& +\left.\frac{4 \Gamma_{L}^{2} \Gamma_{R}^{2}}{\left(\Gamma_{L}^{2}+\Gamma_{R}^{2}\right)^{2}} \frac{\widetilde{\Delta}_{00}^{2}}{\left(\varepsilon-\widetilde{E}_{00}\right)^{2}+\widetilde{\Delta}_{00}^{2}}\right|_{\varepsilon=\mu},
\end{aligned}
$$

where $\Gamma_{\alpha}^{i}=\pi \nu\left|V_{\alpha, i}\right|^{2}$. The resonant widths are $\widetilde{\Delta}_{11} / \widetilde{\Delta}_{0}$ $=\left(2 \cos ^{2} \varphi\right) / 3, \widetilde{\Delta}_{10} / \widetilde{\Delta}_{0}=\left(\cos ^{2} \varphi\right) / 3$, and $\widetilde{\Delta}_{00} / \widetilde{\Delta}_{0}=\sin ^{2} \varphi$ with $\widetilde{\Delta}_{0}=\pi \nu\left|J_{\mathrm{MF}}\langle\vec{\Xi}\rangle\right|^{2}$. The conductance $G$ as a function of $\Delta$ is shown in Fig. 4(b), in a symmetric case of $\Gamma_{L}^{i}=\Gamma_{R}^{i}(i$ $=1,2)$. $G=2 e^{2} / h$ for $\Delta>0$, whereas $G$ goes to zero suddenly for $\Delta<0$. Around $\Delta=0, G$ is larger than the value in the unitary limit, $2 e^{2} / h$, which is attributable to nonuniversal contribution from the multichannel nature of our model. ${ }^{21}$

In the presence of the Zeeman splitting, $E_{1 M}=E_{S=1}$ $-E_{\mathrm{Z}} M$, the resonant level of the triplet state is split into three. With increasing $E_{Z}$, the Kondo effect is rapidly weaken except in the region of $\Delta \sim-E_{Z}$. In Fig. 5(a), we show the Kondo temperature $T_{\mathrm{K}}$ in $E_{\mathrm{Z}}-\Delta$ plane, in the case
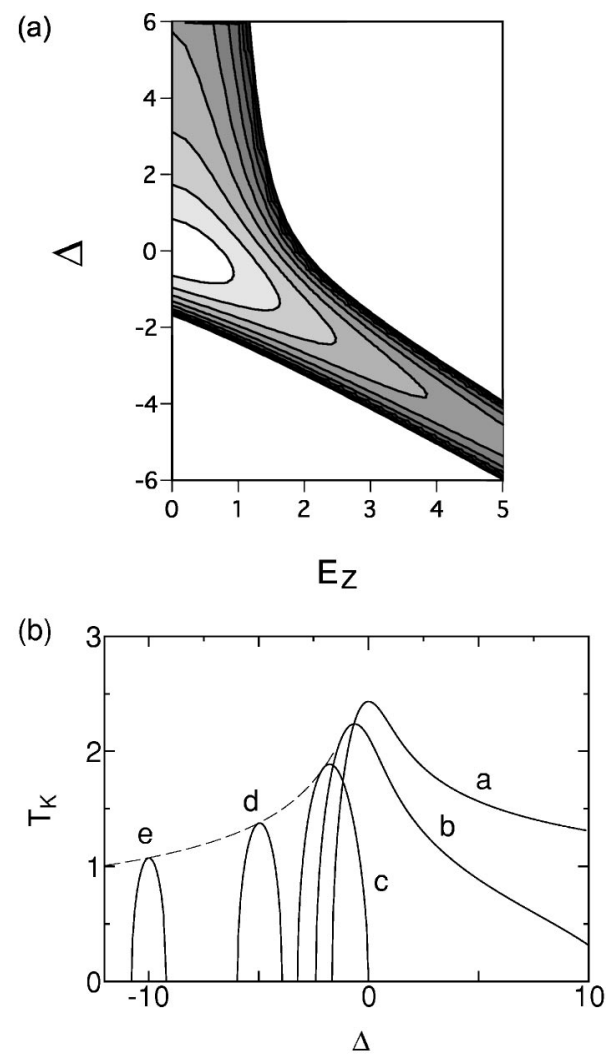

FIG. 5. The mean field calculations for the Kondo temperature $T_{\mathrm{K}}$ in the present model. $\varphi / \pi=0.15$ where $\tan \varphi=\sqrt{3} \widetilde{J} / J_{\mathrm{MF}}$. All of $E_{\mathrm{Z}}, \Delta$, and $T_{\mathrm{K}}$ are in units of $D_{0} \exp \left(-1 / \nu J_{\mathrm{MF}}\right)$. (a) $T_{\mathrm{K}}$ is plotted in $E_{\mathrm{Z}^{-}} \Delta$ plane, by contour lines drawn every 0.25 . The lighter shade indicates the larger values of $T_{\mathrm{K}}$. (b) $T_{\mathrm{K}}$ as a function of $\Delta$ when $E_{\mathrm{Z}}$ is fixed at curve $a, 0$; curve $b, 1$; curve $c, 2$; curve $d, 5$; and curve $e, 10$. The broken line indicates $T_{\mathrm{K}}$ in the case of $-\Delta$ $=E_{\mathrm{Z}}$.

of $\varphi=0.15 \pi$. Figure 5(b) presents $T_{\mathrm{K}}$ as a function of $\Delta$ for several values of $E_{\mathrm{Z}}$. When $E_{\mathrm{Z}}$ is large enough, the Kondo effect takes place only when the resonant state of $|11\rangle$ is overlapped with that of $|00\rangle$. Then $T_{\mathrm{K}}$ is the largest at $\Delta$ $=-E_{\mathrm{Z}}$ and decreases with $\Delta$ being away from this value. At $\Delta=-E_{\mathrm{Z}}, T_{\mathrm{K}}$ obeys a power law

$$
T_{\mathrm{K}}(\Delta)|\Delta|^{1 /\left(2+3 \tan ^{2} \varphi\right)}=\text { const. }
$$

which is indicated by a broken line in Fig. 5(b). This is qualitatively in agreement with the calculated results by the scaling method.

Figure 6 indicates the conductance $G$ in $E_{\mathrm{Z}}-\Delta$ plane, when $\varphi=0.15 \pi$ and $\Gamma_{L}^{i}=\Gamma_{R}^{i}(i=1,2) . G$ takes the value of $2 e^{2} / h$ around $E_{\mathrm{Z}}=0$ and $\Delta>0$, and also along the line of $E_{\mathrm{Z}}=-\Delta .\left(G>2 e^{2} / h\right.$ in the neighborhood of $E_{\mathrm{Z}}=\Delta=0$, as discussed above.) For sufficiently large $E_{Z}$, our model is nearly equivalent to the anisotropic Kondo model with $S$ $=1 / 2{ }^{25,26}$ Hence $G=2 e^{2} / h$ at $\Delta=-E_{\mathrm{Z}}$ and reduces to zero as $\Delta$ deviates from this value, in the same way as in Fig. 3(b) for the case of $S=1 / 2$. 


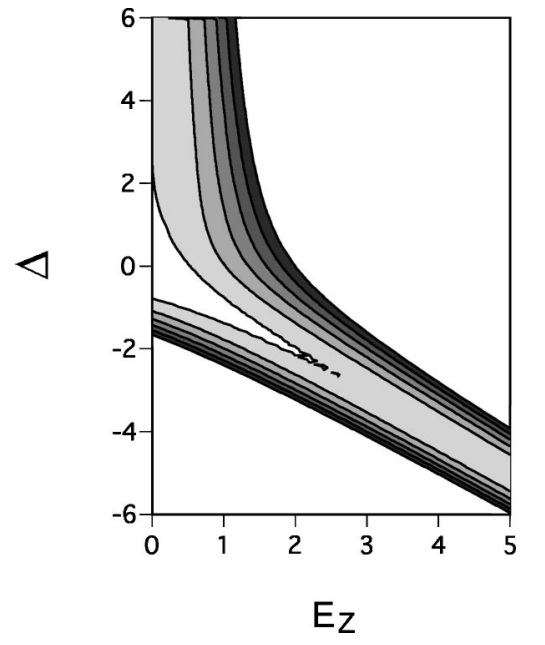

FIG. 6. The mean field calculations for the conductance $G$ in the present model. $G$ is plotted in $E_{\mathrm{Z}^{-}} \Delta$ plane, by contour lines drawn every $0.2\left(2 e^{2} / h\right)$. The lighter shade indicates the larger values of G. $E_{\mathrm{Z}}$ and $\Delta$ are in units of $D_{0} \exp \left(-1 / \nu J_{\mathrm{MF}}\right) . \varphi / \pi=0.15$ where $\tan \varphi=\sqrt{3} \widetilde{J} / J_{\mathrm{MF}}$, and $\Gamma_{L}^{i}=\Gamma_{R}^{i}(i=1,2)$.

\section{CONCLUSIONS AND DISCUSSION}

The Kondo effect in quantum dots with an even number of electrons has been investigated theoretically. The Kondo temperature $T_{\mathrm{K}}$ has been calculated as a function of the energy difference $\Delta=E_{00}-E_{S=1}$ and the Zeeman splitting $E_{\mathrm{Z}}$, using the poor man's scaling method and mean field theory. The scaling calculations have indicated that the competition between the spin-triplet and -singlet states significantly enhances the Kondo effect. When the Zeeman effect is irrelevant, $E_{\mathrm{Z}} \ll T_{\mathrm{K}}, T_{\mathrm{K}}$ is maximal around $\Delta=0$ and decreases with $\Delta$ obeying a power law. In a case of $-\Delta=E_{\mathrm{Z}}$, the Kondo effect takes place from the degeneracy between two states, $|00\rangle$ and $|11\rangle$. Even in this case, the contribution from the other states of higher energy, $|10\rangle$ and $|1-1\rangle$, plays an important role in the enhancement of $T_{\mathrm{K}}$. As a result, $T_{\mathrm{K}}$ is maximal around $E_{\mathrm{Z}}=0$ and depends on $E_{\mathrm{Z}}$ by a power law again.

The mean field theory yields a clear-cut view for the Kondo effect in quantum dots. Considering the spin couplings between the dot states and conduction electrons as a mean field, $\left\langle f_{S M}^{\dagger} c_{k, \sigma}^{(i)}\right\rangle$, we find that the resonant states are created around the Fermi level $\mu$. The resonant width is given by the Kondo temperature $T_{\mathrm{K}}$. The unitary limit of the conductance, $G \sim 2 e^{2} / h$, can be easily understood in terms of the tunneling through these resonant states. In our model, the overlap between the resonant states of $S=1$ and $S=0$ in the dot enhances the Kondo effect. The mean field calculations have led to a power-law dependence of $T_{\mathrm{K}}$ on $\Delta$ and on $E_{\mathrm{Z}}$, in accordance with the scaling calculations.

The mean field theory is not quantitatively accurate for the evaluation of $T_{\mathrm{K}} \cdot{ }^{31}$ (In the case of $S=1 / 2$, the exact value of $T_{\mathrm{K}}$ is obtained accidentally.) In our model, the scaling calculations indicate that all the exchange couplings, $J^{(1)}, J^{(2)}$, and $\widetilde{J}$, are renormalized altogether following Eq. (16) when $|\Delta|$ and $E_{Z}$ are much smaller than the energy scale $D$. In consequence two channels in the leads are coupled effectively for an increase in $T_{\mathrm{K}}$. In the mean field calculations, the interchannel couplings are taken into account in Eq. (37) only partly. In fact, conduction electrons of channel 1 and 2 independently take part in the conductance, Eq. (42). By the perturbation calculations with respect to the exchange couplings, we find that mixing terms between the channels appear in the logarithmic corrections to the conductance. ${ }^{21}$ We could improve the mean field calculations by adopting another form of the order parameter than Eq. (35).

Our calculated results being expressed in terms of $\Delta, E_{\mathrm{Z}}$, and $T_{\mathrm{K}}$ are applicable to any experimental realization, either at small or large magnetic field $B$. At small $B$, our results explain the experimental findings by Sasaki et al.: ${ }^{14}$ The Kondo effect is largely enhanced around $\Delta=0$ when $\Delta$ is tuned by the orbital effect of the magnetic field. $\Delta=0$ at $B$ $\approx 0.2 \mathrm{~T}$, where the Zeeman effect can be safely disregarded. At large $B$, the Zeeman effect splits three components of the spin-triplet state. The Kondo effect has been observed in carbon nanotubes at $B \approx 1 \mathrm{~T}\left(E_{\mathrm{Z}}=g \mu_{\mathrm{B}} B\right.$ with $\left.g=2.0\right)$ where the energy of one component of the triplet state coincides with that of the singlet state. ${ }^{28}$ The Kondo effect induced by the Zeeman effect may be observed in quantum dots on semiconductor heterostructures with a smaller $\mathrm{g}$ factor $(g$ $\approx 0.4$ ), under higher magnetic fields. ${ }^{25,26}$ In such experiments, the value of the Zeeman splitting $E_{\mathrm{Z}}$ can be controlled by applying a large magnetic field parallel to the dot while $\Delta=E_{00}-E_{S=1}$ by a small magnetic field perpendicularly to the dot.

In this paper, we have restricted our considerations to the space of $C_{1}=C_{2}$ in Eq. (4). This is a good starting point in the vicinity of $\Delta=0 .{ }^{35}$ Investigation in the space of $C_{1}$ $=\sqrt{2}$ and $C_{2}=0$, however, has shown different fixed points of the renormalization flow in cases of $E_{\mathrm{Z}}=0^{32}$ and $E_{\mathrm{Z}}$ $=-\Delta \gg T_{\mathrm{K}} \cdot{ }^{25}$ In possible realizations, $C_{2} \approx C_{1}$ for small $|\Delta|$ and $C_{2} \ll C_{1}$ for large $|\Delta|,{ }^{38}$ and our calculations could be incomplete in the latter region. The crossover between the regions requires further studies. A generalized renormalization analysis is in progress for this problem.

\section{ACKNOWLEDGMENTS}

The authors are indebted to L. P. Kouwenhoven, S. De Franceschi, J. M. Elzerman, K. Maijala, S. Sasaki, W. G. van der Wiel, Y. Tokura, L. I. Glazman, M. Pustilnik, and G. E. W. Bauer for valuable discussions. The authors acknowledge financial support from the "Netherlandse Organisatie voor Wetenschappelijk Onderzoek" (NWO). M. E. is also grateful for financial support from the Japan Society for the Promotion of Science for his stay at Delft University of Technology.

\section{APPENDIX A: MEAN FIELD CALCULATIONS FOR $S=1 / 2$}

The original Hamiltonian for a quantum dot with one energy level reads

$$
\begin{aligned}
H= & \sum_{\alpha=L, R} \sum_{k \sigma} \varepsilon_{k} c_{\alpha, k \sigma}^{\dagger} c_{\alpha, k \sigma}+\sum_{\alpha=L, R} \sum_{k \sigma}\left(V_{\alpha} c_{\alpha, k \sigma}^{\dagger} d_{\sigma}+\text { H.c. }\right) \\
& +H_{\mathrm{dot}}
\end{aligned}
$$


with

$$
H_{\mathrm{dot}}=\sum_{\sigma} E_{0} d_{\sigma}^{\dagger} d_{\sigma}+U d_{\uparrow}^{\dagger} d_{\uparrow} d_{\downarrow}^{\dagger} d_{\downarrow}
$$

For the state of one electron in the dot, the addition and extraction energies are given by $E^{+}=E_{0}+U-\mu$ and $E^{-}$ $=\mu-E_{0}$, respectively. The parameters, $E_{0}$ and $U$, in Eq. (A2) should be determined to fit these energies to experimental data. For conduction electrons in leads $L, R$, we perform a unitary transformation, $c_{k \sigma}=\left(V_{L}^{*} c_{L, k \sigma}+V_{R}^{*} c_{R, k \sigma}\right) / V, \bar{c}_{k \sigma}$ $=\left(-V_{R, i} c_{L, k \sigma}+V_{L, i} c_{R, k \sigma}\right) / V$, with $\quad V=\sqrt{\left|V_{L}\right|^{2}+\left|V_{R}\right|^{2}}$, along the lines of Ref. 9. We disregard the modes $\bar{c}_{k \sigma}$ that are uncoupled to the quantum dot.

We consider the Coulomb blockade region for one electron, where both $E^{+}$and $E^{-}$are much larger than the level broadening $\Gamma=\pi \nu V^{2}$ ( $\nu$ being the density of states in the leads) and temperature. Integrating out the dot states with zero or two electrons by the Schrieffer-Wolff transformation, ${ }^{7,8}$ we obtain the effective low-energy Hamiltonian

$$
\begin{aligned}
H= & \sum_{k \sigma} \varepsilon_{k} c_{k \sigma}^{\dagger} c_{k \sigma}+\sum_{\sigma} E_{\sigma} f_{\sigma}^{\dagger} f_{\sigma}+J \sum_{k k^{\prime}}\left[\hat{S}_{+} c_{k^{\prime} \downarrow}^{\dagger} c_{k \uparrow}\right. \\
& \left.+\hat{S}_{-} c_{k^{\prime} \uparrow}^{\dagger} c_{k \downarrow}+\hat{S}_{z}\left(c_{k^{\prime} \uparrow}^{\dagger} c_{k \uparrow}-c_{k^{\prime} \downarrow}^{\dagger} c_{k \downarrow}\right)\right]
\end{aligned}
$$

under a constraint of Eq. (29). In the second term we have included the Zeeman effect, $E_{\uparrow, \downarrow}=E_{0} \pm E_{\mathrm{Z}}$. The third term represents the exchange coupling between the dot spin and conduction electrons with $J=V^{2} / E_{\mathrm{c}}$, where $1 / E_{\mathrm{c}}=1 / E^{+}$ $+1 / E^{-}$. By expressing the spin operator $\hat{S}$ as $\hat{S}_{+}=f_{\uparrow}^{\dagger} f_{\downarrow}$, $\hat{S}_{-}=f_{\downarrow}^{\dagger} f_{\uparrow}, \hat{S}_{z}=\left(f_{\uparrow}^{\dagger} f_{\uparrow}-f_{\downarrow}^{\dagger} f_{\downarrow}\right) / 2$, one finds that Eq. (A3) is identical to Eq. (28).

The mean field Hamiltonian, Eq. (31), includes "energy levels" for pseudofermions, $\widetilde{E}_{\sigma}=E_{\sigma}+\lambda$, which are coupled to the leads with "tunneling amplitude," $\widetilde{V}=-\sqrt{2} J\langle\Xi\rangle$. The Green function for the pseudofermions is

$$
G_{\sigma}(\varepsilon)=\frac{1}{\varepsilon-\widetilde{E}_{\sigma}+\mathrm{i} \widetilde{\Delta}},
$$

where $\widetilde{\Delta}=\pi \nu|\widetilde{V}|^{2}$. This represents the resonant tunneling with the resonant width $\tilde{\Delta}$.

The expectation value of the Hamiltonian, Eq. (31), is written as

$E_{\mathrm{MF}}=\sum_{\sigma}\left[-\frac{\widetilde{\Delta}}{\pi}+\frac{\widetilde{E}_{\sigma}}{\pi} \tan ^{-1} \frac{\tilde{\Delta}}{\widetilde{E}_{\sigma}}+\frac{\widetilde{\Delta}}{2 \pi} \ln \frac{\widetilde{E}_{\sigma}^{2}+\widetilde{\Delta}^{2}}{D_{0}^{2}}\right]-\lambda+\frac{\widetilde{\Delta}}{\pi \nu J}$,

where $D_{0}$ is the bandwidth in the leads. ${ }^{7}$ We set $\mu=0$ in this appendix. The constraint of Eq. (29) is equivalent to the condition

$$
\frac{\partial E_{\mathrm{MF}}}{\partial \lambda}=\frac{1}{\pi} \sum_{\sigma} \tan ^{-1} \frac{\widetilde{\Delta}}{\widetilde{E}_{\sigma}}-1=0
$$

This yields $E_{0}+\lambda=0$. The minimization of $E_{\mathrm{MF}}$ with respect to $\tilde{\Delta}$ (or $|\langle\Xi\rangle|^{2}$ ) determines $\tilde{\Delta}$

$$
\frac{\partial E_{\mathrm{MF}}}{\partial \widetilde{\Delta}}=\frac{1}{2 \pi} \sum_{\sigma} \ln \frac{\widetilde{E}_{\sigma}^{2}+\widetilde{\Delta}^{2}}{D_{0}^{2}}+\frac{1}{\pi \nu J}=0 .
$$

For $E_{\mathrm{Z}}=0$, we find

$$
\widetilde{\Delta}=D_{0} \exp [-1 / 2 \nu J] \equiv \widetilde{\Delta}_{0} .
$$

This is equal to the Kondo temperature, $T_{\mathrm{K}}$. For $E_{\mathrm{Z}} \neq 0$, Eq. (A7) yields

$$
\widetilde{\Delta}^{2}+E_{\mathrm{Z}}^{2}=\widetilde{\Delta}_{0}^{2}
$$

Using the $T$ matrix, $\hat{T}$, the conductance through the dot, $G$, is given by

$$
\begin{aligned}
G= & \left.\frac{e^{2}}{h}(2 \pi \nu)^{2} \sum_{\sigma}\left|\left\langle R, k^{\prime} \sigma|\hat{T}| L, k \sigma\right\rangle\right|^{2}\right|_{\varepsilon_{k}=\varepsilon_{k^{\prime}}=\mu} \\
= & \frac{e^{2}}{h}(2 \pi \nu)^{2} \frac{\left|V_{L}\right|^{2}\left|V_{R}\right|^{2}}{\left(\left|V_{L}\right|^{2}+\left|V_{R}\right|^{2}\right)^{2}} \\
& \times\left.\sum_{\sigma}\left|\left\langle\psi_{k^{\prime} \sigma}|\hat{T}| \psi_{k \sigma}\right\rangle\right|^{2}\right|_{\varepsilon_{k}=\varepsilon_{k^{\prime}}=\mu} \\
= & \left.\frac{e^{2}}{h} \frac{4 \Gamma_{L} \Gamma_{R}}{\left(\Gamma_{L}+\Gamma_{R}\right)^{2}} \sum_{\sigma} \frac{\widetilde{\Delta}^{2}}{\left(\varepsilon-\widetilde{E}_{\sigma}\right)^{2}+\widetilde{\Delta}^{2}}\right|_{\varepsilon=\mu},
\end{aligned}
$$

where $\Gamma_{\alpha}=\pi \nu\left|V_{\alpha}\right|^{2}$. This yields Eq. (34) in the text. On the second line in Eq. (A10), $\left|\psi_{k \sigma}\right\rangle=c_{k \sigma}^{\dagger}|0\rangle=\left(V_{L}|L, k \sigma\rangle\right.$ $\left.+V_{R}|R, k \sigma\rangle\right) / V$, and the $T$ matrix is evaluated in terms of the Green function, Eq. (A4), $|\widetilde{V}|^{2} G_{\sigma}\left(\varepsilon=\varepsilon_{k}\right)$.

\section{APPENDIX B: MEAN FIELD CALCULATIONS IN THE PRESENT MODEL}

For the spin states of the coupling between the spin triplet $S=1$ in the dot and a conduction electron, we introduce spinors of $S=1 / 2$ and $3 / 2$. Using the Clebsch-Gordan coefficients, they are given by

$$
\begin{gathered}
\vec{\Omega}_{1 / 2}^{(i)}=\sum_{k}\left(\begin{array}{c}
\left(\sqrt{2} f_{11}^{\dagger} c_{k \uparrow}^{(i)}+f_{10}^{\dagger} c_{k \downarrow}^{(i)}\right) / \sqrt{3} \\
\left(\sqrt{2} f_{1-1}^{\dagger} c_{k \downarrow}^{(i)}+f_{10}^{\dagger} c_{k \uparrow}^{(i)}\right) / \sqrt{3}
\end{array}\right), \\
\vec{\Omega}_{3 / 2}^{(i)}=\sum_{k}\left(\begin{array}{c}
f_{11}^{\dagger} c_{k \downarrow}^{(i)} \\
\left(-f_{11}^{\dagger} c_{k \uparrow}^{(i)}+\sqrt{2} f_{10}^{\dagger} c_{k \downarrow}^{(i)}\right) / \sqrt{3} \\
\left(f_{1-1}^{\dagger} c_{k \downarrow}^{(i)}-\sqrt{2} f_{10}^{\dagger} c_{k \uparrow}^{(i)}\right) / \sqrt{3} \\
-f_{1-1}^{\dagger} c_{k \uparrow}^{(i)}
\end{array}\right) .
\end{gathered}
$$

The exchange couplings between the triplet state and conduction electrons, Eq. (12), can be rewritten as

$$
H^{S=1}=\sum_{i=1,2} J^{(i)}\left[-2 \vec{\Omega}_{1 / 2}^{(i) \dagger} \vec{\Omega}_{1 / 2}^{(i)}+\vec{\Omega}_{3 / 2}^{(i) \dagger} \vec{\Omega}_{3 / 2}^{(i)}\right]
$$


In the same way we define the spinors of $S=1 / 2$ to represent the spin couplings between the singlet state $S=0$ and a conduction electron

$$
\vec{\Psi}^{(i)}=\sum_{k}\left(\begin{array}{c}
f_{00}^{\dagger} c_{k \downarrow}^{(\bar{i})} \\
-f_{00}^{\dagger} c_{k \uparrow}^{(\bar{i} \uparrow}
\end{array}\right),
$$

where $\bar{i}=2$ and 1 for $i=1$ and 2 , respectively. The conversion between the triplet and singlet states, Eq. (13), is rewritten as

$$
H^{S=1 \leftrightarrow 0}=-\sqrt{3} \widetilde{J} \sum_{i=1,2}\left[\vec{\Psi}^{(i) \dagger} \vec{\Omega}_{1 / 2}^{(i)}+\text { H.c. }\right] .
$$

In $H^{S=1}+H^{S=1 \leftrightarrow 0}$, a mode of the largest coupling with $S$ $=1 / 2$ is given by

$$
\vec{\Xi}=(\cos \varphi) \vec{\Omega}_{1 / 2}^{(1)}+(\sin \varphi) \vec{\Psi}^{(1)}
$$

for $J^{(1)} \geqslant J^{(2)}$, which is Eq. (35) in the text. The corresponding eigenvalue is given by Eq. (37) and $\varphi$ is determined as in Eq. (38).

The mean field Hamiltonian, Eq. (36), represents the resonant tunneling through the energy levels for the pseudofermions, $\widetilde{E}_{S M}=E_{S M}+\lambda$. The expectation value of Eq. (36), $E_{\mathrm{MF}}$, is evaluated in the same way as in Appendix A. $\partial E_{\mathrm{MF}} / \partial \lambda=0$ yields

$$
\tan ^{-1} \frac{\widetilde{\Delta}_{11}}{\widetilde{E}_{11}}+\tan ^{-1} \frac{\widetilde{\Delta}_{10}}{\widetilde{E}_{10}}+\tan ^{-1} \frac{\widetilde{\Delta}_{00}}{\widetilde{E}_{00}}=\pi,
$$

where the resonant widths are $\widetilde{\Delta}_{11} / \widetilde{\Delta}_{0}=\left(2 \cos ^{2} \varphi\right) / 3$, $\widetilde{\Delta}_{10} / \widetilde{\Delta}_{0}=\left(\cos ^{2} \varphi\right) / 3$, and $\widetilde{\Delta}_{00} / \widetilde{\Delta}_{0}=\sin ^{2} \varphi$ with $\widetilde{\Delta}_{0}$ $=\pi \nu\left|J_{\mathrm{MF}}\langle\vec{\Xi}\rangle\right|^{2}$. We set $\mu=0$ here. Minimizing $E_{\mathrm{MF}}$ with respect to $\widetilde{\Delta}_{0}$, we obtain

$$
\begin{gathered}
\frac{2}{3}\left(\cos ^{2} \varphi\right) \ln \frac{\widetilde{E}_{11}^{2}+\widetilde{\Delta}_{11}^{2}}{D_{0}^{2}}+\frac{1}{3}\left(\cos ^{2} \varphi\right) \ln \frac{\widetilde{E}_{10}^{2}+\widetilde{\Delta}_{10}^{2}}{D_{0}^{2}} \\
+\left(\sin ^{2} \varphi\right) \ln \frac{\widetilde{E}_{00}^{2}+\widetilde{\Delta}_{00}^{2}}{D_{0}^{2}}+\frac{2}{\nu J}=0 .
\end{gathered}
$$

Equations (B7) and (B8) determine $\lambda$ and $\widetilde{\Delta}_{0}$ (or $|\langle\vec{\Xi}\rangle|^{2}$ ). The conductance through the dot is given by

$$
\begin{aligned}
G= & \left.\frac{e^{2}}{h}(2 \pi \nu)^{2} \sum_{i, j, \sigma, \sigma^{\prime}}\left|\left\langle R, k^{\prime} \sigma^{\prime}, j|\hat{T}| L, k \sigma, i\right\rangle\right|^{2}\right|_{\varepsilon_{k}=\varepsilon_{k^{\prime}}=\mu} \\
= & \frac{e^{2}}{h}(2 \pi \nu)^{2} \sum_{i, j, \sigma, \sigma^{\prime}} \frac{\Gamma_{R}^{j}}{\Gamma_{L}^{j}+\Gamma_{R}^{j}} \frac{\Gamma_{L}^{i}}{\Gamma_{L}^{i}+\Gamma_{R}^{i}} \\
& \times\left.\left|\left\langle\psi_{k^{\prime} \sigma^{\prime}}^{(j)}|\hat{T}| \psi_{k \sigma}^{(i)}\right\rangle\right|^{2}\right|_{\varepsilon_{k}=\varepsilon_{k^{\prime}}=\mu},
\end{aligned}
$$

where $\quad \Gamma_{\alpha}^{i}=\pi \nu\left|V_{\alpha, i}\right|^{2} \quad$ and $\quad\left|\psi_{k \sigma}^{(i)}\right\rangle=\left(V_{L, i}|L, k \sigma, i\rangle\right.$ $\left.+V_{R, i}|R, k \sigma, i\rangle\right) / V_{i}$. The $T$ matrix can be evaluated, using the Green function for the pseudofermions, $G_{S M}(\varepsilon)=[\varepsilon$ $\left.-\widetilde{E}_{S M}+\mathrm{i} \widetilde{\Delta}_{S M}\right]^{-1}$, as in Appendix A. This yields Eq. (42) in the text.
${ }^{1}$ D. Goldhaber-Gordon, H. Shtrikman, D. Mahalu, D. AbuschMagder, U. Meirav, and M.A. Kastner, Nature (London) 391, 156 (1998); D. Goldhaber-Gordon, J. Göres, M.A. Kastner, H. Shtrikman, D. Mahalu, and U. Meirav, Phys. Rev. Lett. 81, 5225 (1998).

${ }^{2}$ S.M. Cronenwett, T.H. Oosterkamp, and L.P. Kouwenhoven, Science 281, 540 (1998).

${ }^{3}$ F. Simmel, R.H. Blick, J.P. Kotthaus, W. Wegscheider, and M. Bichler, Phys. Rev. Lett. 83, 804 (1999).

${ }^{4}$ J. Schmid, J. Weis, K. Eberl, and K.v. Klitzing, Phys. Rev. Lett. 84, 5824 (2000).

${ }^{5}$ W.G. van der Wiel, S. De Franceschi, T. Fujisawa, J.M. Elzerman, S. Tarucha, and L.P. Kouwenhoven, Science 289, 2105 (2000).

${ }^{6}$ J. Kondo, Prog. Theor. Phys. 32, 37 (1964).

${ }^{7}$ A. C. Hewson, The Kondo Problem to Heavy Fermions (Cambridge, Cambridge, England, 1993).

${ }^{8}$ K. Yosida, Theory of Magnetism (Springer, New York, 1996).

${ }^{9}$ L.I. Glazman and M.É. Răkh, Pis'ma Zh. Éksp. Teor. Fiz. 47, 378 (1988) [ JETP Lett. 47, 452 (1988)].

${ }^{10}$ T.K. Ng and P.A. Lee, Phys. Rev. Lett. 61, 1768 (1988).

${ }^{11}$ A. Kawabata, J. Phys. Soc. Jpn. 60, 3222 (1991).

${ }^{12}$ S. Hershfield, J.H. Davies, and J.W. Wilkins, Phys. Rev. Lett. 67, 3720 (1991); Phys. Rev. B 46, 7046 (1992).

${ }^{13}$ Y. Meir, N.S. Wingreen, and P.A. Lee, Phys. Rev. Lett. 70, 2601 (1993).
${ }^{14}$ S. Sasaki, S. De Franceschi, J.M. Elzerman, W.G. van der Wiel, M. Eto, S. Tarucha, and L.P. Kouwenhoven, Nature (London) 405, 764 (2000).

${ }^{15}$ S. Tarucha, D.G. Austing, T. Honda, R.J. van der Hage, and L.P. Kouwenhoven, Phys. Rev. Lett. 77, 3613 (1996).

${ }^{16}$ L.P. Kouwenhoven, T.H. Oosterkamp, M.W.S. Danoesastro, M. Eto, D.G. Austing, T. Honda, and S. Tarucha, Science 278, 1788 (1997).

${ }^{17}$ T. Inoshita, A. Shimizu, Y. Kuramoto, and H. Sakaki, Phys. Rev. B 48, R14725 (1993); T. Inoshita, Y. Kuramoto, and H. Sakaki, Superlattices Microstruct. 22, 75 (1997).

${ }^{18}$ T. Pohjola, J. König, M.M. Salomaa, J. Schmid, H. Schoeller, and G. Schön, Europhys. Lett. 40, 189 (1997).

${ }^{19}$ W. Izumida, O. Sakai, and Y. Shimizu, J. Phys. Soc. Jpn. 67, 2444 (1998).

${ }^{20}$ A. Levy Yeyati, F. Flores, and A. Martín-Rodero, Phys. Rev. Lett. 83, 600 (1999).

${ }^{21}$ M. Eto and Yu.V. Nazarov, Phys. Rev. Lett. 85, 1306 (2000).

${ }^{22}$ P.W. Anderson, J. Phys. C 3, 2436 (1970).

${ }^{23}$ P. Nozières and A. Blandin, J. Phys. (Paris) 41, 193 (1980).

${ }^{24}$ D.L. Cox and A. Zawadowski, Adv. Phys. 47, 599 (1998).

${ }^{25}$ M. Pustilnik, Y. Avishai, and K. Kikoin, Phys. Rev. Lett. 84, 1756 (2000).

${ }^{26}$ D. Giuliano and A. Tagliacozzo, Phys. Rev. Lett. 84, 4677 (2000). 
${ }^{27}$ L. P. Kouwenhoven (private communications).

${ }^{28}$ J. Nygård, D.H. Cobden, and P.E. Lindelof, Nature (London) 408, 342 (2000).

${ }^{29}$ A. Yoshimori and A. Sakurai, Suppl. Prog. Theor. Phys. 46, 162 (1970)

${ }^{30}$ C. Lacroix and M. Cyrot, Phys. Rev. B 20, 1969 (1979).

${ }^{31}$ This mean field theory is equivalent to the mean field theory using the slave bosons for $U=\infty$ Anderson model in the Kondo region (Ref. 7). This method is exact in the large- $N$ limit when the dot state is $N$-fold degenerate.

${ }^{32}$ M. Pustilnik and L.I. Glazman, Phys. Rev. Lett. 85, 2993 (2000).

${ }^{33}$ I. Affleck, A.W.W. Ludwig, and B.A. Jones, Phys. Rev. B 52, 9528 (1995).

${ }^{34}$ The different symmetry means different orbital quantum number for the one-electron states in a quantum dot. We consider the situation for vertical quantum dots where the orbital quantum number $i$ is conserved in the tunneling processes between the dot and leads.

${ }^{35}$ One finds that $C_{1}=C_{2}=1$ in the case of $\delta=0$, considering the matrix element of the Coulomb interaction between $d_{1 \uparrow}^{\dagger} d_{1 \downarrow}^{\dagger}|0\rangle$ and $d_{2 \uparrow}^{\dagger} d_{2 \downarrow}^{\dagger}|0\rangle,\left\langle 22\left|e^{2} / r\right| 11\right\rangle \equiv K$. In rectangular dots (Ref. 14), the matrix element $K$ is not zero and of the same order as the exchange interaction, $\left\langle 12\left|e^{2} / r\right| 21\right\rangle \equiv J$, and smaller than the Hartree term, $\left\langle 11\left|e^{2} / r\right| 11\right\rangle \sim$ (charging energy), typically by one order. In a case of $\left\langle 11\left|e^{2} / r\right| 11\right\rangle=\left\langle 22\left|e^{2} / r\right| 22\right\rangle, C_{1} / C_{2}=(\delta$ $+\sqrt{\delta^{2}+K^{2}}$ ) $/ K$ and thus $C_{1} \approx C_{2}$ for $\delta \sim K$ (when the singlet and triplet states are degenerate, $\delta \sim J)$.

${ }^{36}$ F.D.M. Haldane, J. Phys. C 11, 5015 (1978).

${ }^{37}$ I. Okada and K. Yosida, Prog. Theor. Phys. 49, 1483 (1973).

${ }^{38}$ This result is different from that in Ref. 25, which treats the situation of $C_{1}=\sqrt{2}, C_{2}=0$ in Eq. (4). Their calculations might be better when $|\Delta|=E_{\mathrm{Z}}$ is so large that $\delta \gg K$ (Ref. 35) $(|\Delta|$ increases with $\delta$ ). See discussion in Sec. V. 